\title{
Anaerobe Glykolyse bei Ehrlich-Aszites-Tumorzellen
}

\author{
1. Mitteilung: Beeinflussung durch Adenosin-(mono-, di-, tri-) phosphat solvie Nicotinsäureamid
}

\author{
Von W. Lührs') und K. Chrometzka \\ Aus dem Sanatorium „Bergfrieden“ - Rottach-Egern der Arbeitsgemeinschaft für Krebsbekämpfung Sitz Bochum - \\ Rubrknappschaft (Chefarzt: Prof. Dr. W. Lübrs)
}

(Der Schriftleitung zugegangen am 16. Dezember 1963)

\begin{abstract}
Eine Zugabe von FDP-ase zu Ehrlich-Aszites-Tumorzellen ließ eine deutliche Glykolysehemmung erkennen. Die Hemmwirkung stand in einer gewissen Beziehung zur Aktivität dieser Fermentlösung.

Bei Zusatz von AMP, ADP und ATP ließen sich unterschiedliche Wirkungen auf die anaetobe Glykolyse von Ehrlich-Aszites-Turmorzellen in Kombination mit FDP-ase feststellen. Der Einfluß steigender Konzentrationen von AMP auf reine Aszites-Tumorzellen ergab eine Hemmung der Glykolyse. AMP und FDP-ase ergaben zusammen eine stärkere Hemmung der Glykolyse als FDP-ase allein. Zugabe von ADP zu reinen Asziteszellen sowohl allein als auch in Kombination mit FDP-ase beeinflußte die Glykolyse nicht. Steigende Konzentrationen von ATP förderten die Glykolyse reiner Asziteszellen. Die hemmende Wirkung der FDP-ase wurde durch gleichzeitige Zugabe von $12 \mu \mathrm{Mol}$ ATP/Ansatz zum Teil aufgehoben. Nicotinsäureamid hatte, auch in Kombination mit FDP-ase, keinen Einfluß auf die Glykolyse von Asziteszellen.
\end{abstract}

Glycolysis in Ehrlich ascites tumour cells is markedly inhibited by the addition of FDP-ase. The degree of inhibition is proportional to the activity of the FDP-ase solution.

AMP, ADP or ATP, with and without FDP-ase, have different effects on the anaerobic glycolysis of Ehrlich ascites tumour cells. Increasing concentrations of AMP cause an inhibition of glycolysis in pure ascites tumour cells. AMP and FDP-ase inhibit more strongly than FDP-ase alone. The addition of ADP alone or with FDP-ase to pure ascites cells does not affect glycolysis. Increasing concentrations of ATP promote glycolysis in pure ascites cells. The inhibitory action of FDP-ase is partly abolished by the simultaneous addition of $12 \mu$ mole ATP per experiment. Nicotinamide, even with FDP-ase, had no effect on the glycolysis of ascites cells.

In einer Mitteilung (1) hatten wir innerhalb des Abbauweges der Glukose auch die Umkehr bis zur Glukoneogenese und die Nebenwege des Glukose-PhosphatShunt's (WARBURG, DiCKENS) und einen halboxydativen Kurzschlußmechanismus nach DoudorofF und ENTNER aufgezeigt. Gerade die Umkehr der Glykolyse, die auf ihrem rückläufigen Weg bis zur Neubildung von Zucker von der Peripherie aus gesteuert wird, gewinnt in neuerer Zeit immer mehr an Interesse.

1963 berichtete H. A. KREBS auf der Nobelpreisträgertagung in Lindau Einzelheiten über enzymatische Regelungsmechanismen innerhalb der Neubildung von Zucker im Tierkörper (2). Eine Zuckerneubildung oder Glukoneogenese aus Milchsäure, Propionsäure, Aminosäuren, Citronensäure oder anderen Intermediärprodukten des Citronensäurecyklus, ist nach seiner Meinung ein biosynthetischer Stoffwechselvorgang von großer Bedeutung, sowohl qualitativ als auch quantitativ. Wir hatten früher darauf hingewiesen, daß die Dephosphory-

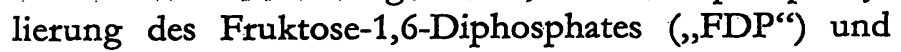
des Glukose-6-Phosphates („G6P“) durch die substratspezifischen Enzyme keine Umkehrungen einfacher Phosphorylierungsteaktionen darstellen können. Nach H. A. KREBS wird auch die Aktivität der Fruktose-1,6Diphosphatase durch spezifische Rückkopplungsmechanismen reguliert. Auf Grund der energetischen Verhältnisse erscheint das Ferment. FDP-ase in seiner Kinetik ungewöhnlich. KREBS fand, daß dieses Ferment durch höhere Substratkonzentrationen (über $1 \mathrm{mMol}$ ) gehemmt wird, wenn Adenosinmonophosphat anwesend ist.

1) Meinem Lehrer, Herrn Professor K. Tromas, zum 80. Geburtstag in dankbarer Verehrung gewidmet.
Bekanntlich wird nach WARBURG (3) in der Tumorzelle sehr viel mehr Zucker abgebaut. Die Glykolyse ist in der Karzinomzelle vorherrschend. Eine Resynthese von Glukose ist kaum nachzuweisen. Nach WeBer und CANTERo (4) konnte im malignen Hepatom bei Ratten die Glukose-6-Phosphatase ("G6P-ase") nicht gefunden werden. Weber, Ashmore und Cantero (5) konnten auch Fruktose-1,6-Diphosphatase („FDP-ase") unter gleichen Versuchsbedingungen in nennenswerter Aktivität nicht nachweisen. Dieses Ferment wurde 1933 von LoHmanN (6) entdeckt und war unter dem Namen Hexose-diphosphatase bekannt. Bereits 1950 konnten Roche und Bouchilloux (7) nachweisen, daß die FDP-ase, damals noch oft Hexose-diphosphatase genannt, ihre spezifische Aufgabe erfüllt, Fruktose-1,6Diphosphat zu dephosphorylieren.

Unsere eigenen Untersuchungen in der Leber von krebskranken Patienten - bei diagnostischen Laparoskopien oder inter operationem - bestätigen bis zu einem gewissen Grade die Befunde der experimentellen Krebsforschung (8). Bereits 1960 hatten wir den Einfluß dieses Fermentes auf die Glykolyse von Ehrlich-Aszites-

\footnotetext{
Abkürzungen:

FDP-ase $=$ Fruktose-1,6-Diphosphatase PGI $=$ Phosphoglukoseisomerase

G-6-P-DH = Glukose-6-phosphatdehydrogenase

TPN $=$ Triphosphopyridinnucleotid

TRA $=$ Triäthanolamin

FDP = Fruktose-1,6-Diphosphat

AMP $=$ Adenosinmonophosphat

ADP $=$ Adenosindiphosphat

$\mathrm{ATP}=$ Adenosintriphosphat.
} 
Tumorzellen geprüft und eine etwa 60-proz. Hemmung der Gärung in vitro erreichen können. Eine gleichzeitige Beeinflussung der Atmung in der Krebszelle in positivem oder negativem Sinne ließ sich nicht nachweisen. In neuerer Zeit hatten wir wiederum die Einwirkung einer Fermentlösung, die aus frischer Kaninchenleber gewonnen wurde, nachgeprüft.

\section{Versuche}

\section{Versucbsmaterial}

Die Versuche wurden mit Ehrlich-Aszites-Tumorzellen durchgeführt. Weiße Mäuse (NMRI-Stamm) wurden mit $0,4 \mathrm{ml}$ Aszitessuspension intraperitoneal implantiert. Nach 8-10 Tagen wurden die Mäuse dekapiert, die Aszitessuspension entnommen und zentrifugiert. Die Zellen wurden mehrmals mit Ringerlösung gewaschen und auf etwa $20 \mathrm{mg}$ Trockengewicht $/ \mathrm{m} /$ Ringerlösung verdünnt. Falls nichts anderes bemerkt, wurden jeweils $0,3 \mathrm{ml}$ Aszitessuspension in die Gefäße einpipettiert.

\section{Messung der Glykolyse}

Es wurde die anaerobe Glykolyse der Ehrlich-Aszites-Tumorzellen unter Zusatz von FDP-ase untersucht. Die anaerobe Glykolyse wurde nach der von WARBuRg (3) angegebenen Methode in einem Medium aus Ringerlösung, Glukose und Bikarbonat gemessen. In den Hauptraum der Gefäße wurden neben RingerBikarbonat-Glukoselösung und Asziteszellen unterschiedliche Mengen einer aktiven Fermentlösung zugegeben. Der Gasraum enthielt $5 \% \quad \mathrm{CO}_{2}$ in $95 \% \mathrm{~N}_{2}$. Die Inkubationszeit betrug 60 Minuten, die Temperatur $37^{\circ}$. Die Endkonzentration an Glukose betrug für jedes Gefäß 34,0 $\mu \mathrm{Mol}$.

Isolierung der FDP-ase

Die FDP-ase wurde in den Bayerwerken aus frischer Kaninchenleber nach der Methode von Lewis, Mokrasch und McGirvery (9) hergestellt. Die Eiweißkonzentration der Fermentlösung betrug im Durchschnitt 20-30 mg Protein $/ \mathrm{m} /$. Zwei Fermentaufbereitungen wiesen eine Eiweißkonzentration von etwa $60-90$ $\mathrm{mg} / \mathrm{m} l$ Enzymlösung auf. $0,1-0,4 \mathrm{~m} l$ der Fermentlösung wurden für die Versuche eingesetzt.

\section{Aktivitätsbestimmung}

Die Aktivität der Fermentlösung wurde im optischen Test nach folgenden Reaktionen bestimmt:

Fructose-1,6-Diphosphat

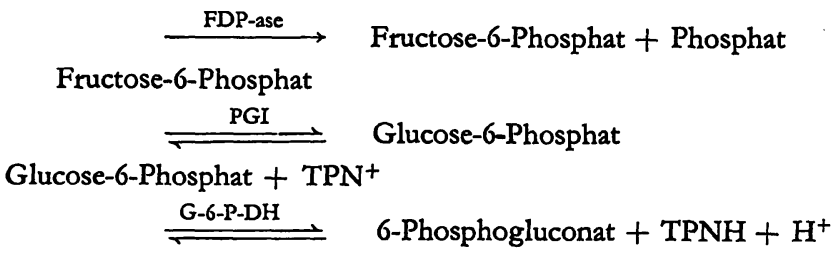

wobei die Zunahme der TPNH-Extinktion bei $366 \mathrm{~m} \mu$ als Meßgröße dient. Pro $\mu \mathrm{Mol}$ FDP entsteht ein $\mu \mathrm{Mol}$ TPNH.

\section{Ansatz (Mengen in $\mathrm{ml}$ )}

1,94 TRA-Puffer $0,05 \mathrm{~m}$; 0,30 FDP-Cyclohexylammoniumsalz $0,05 \mathrm{~m} ; 0,30 \mathrm{MgCl}_{2} \cdot 6 \mathrm{H}_{2} \mathrm{O}, 0,05 \mathrm{~m} ; 0,30 \mathrm{MnSO}_{4}, 0,05 \mathrm{~m} ; 0,10$ TPN (10 mg/ml), 0,02 PGI (Boehringer) (15,6 E); 0,02 G-6-P-DH (Boehringer) (2,8 E); 0,02 Fermentlösung.

Die Extinktion bei $366 \mathrm{~m} \mu$ wurde in $\operatorname{der} 1 \mathrm{~cm}$-Küvette bei Zimmertemperatur über 10 Minuten bestimmt und daraus die Enzymaktivität berechnet. Die Enzymaktivität wird in Milli-Einheiten/mg Protein angegeben:

1 Milli-Einheit $=1 / 1000 \mathrm{IE} ; 1 \mathrm{IE}=1 \mu \mathrm{Mol} / \mathrm{Min}$. Substratumsatz entspricht einer Extinktionsänderung von $\Delta \mathrm{E} / \Delta \mathrm{t}=1,1 / \mathrm{Min}$. in $3,0 \mathrm{ml}$

1 Milli-Einheit $=\Delta \mathrm{E} 0,0011366 \mathrm{~m} \mu / \mathrm{Min} . \cdot 3 \mathrm{ml}$.

Die -Proteinbestimmung erfolgte mit dem Biuret-Reagenz.

\section{Ergebnisse}

Die Untersuchungen mit dieser Fermentlösung ließen wiederum eine deutliche Hemmung der Glykolyse bei Ehrlich-Aszites-Tumorzellen in vitro in der WarburgApparatur erkennen.

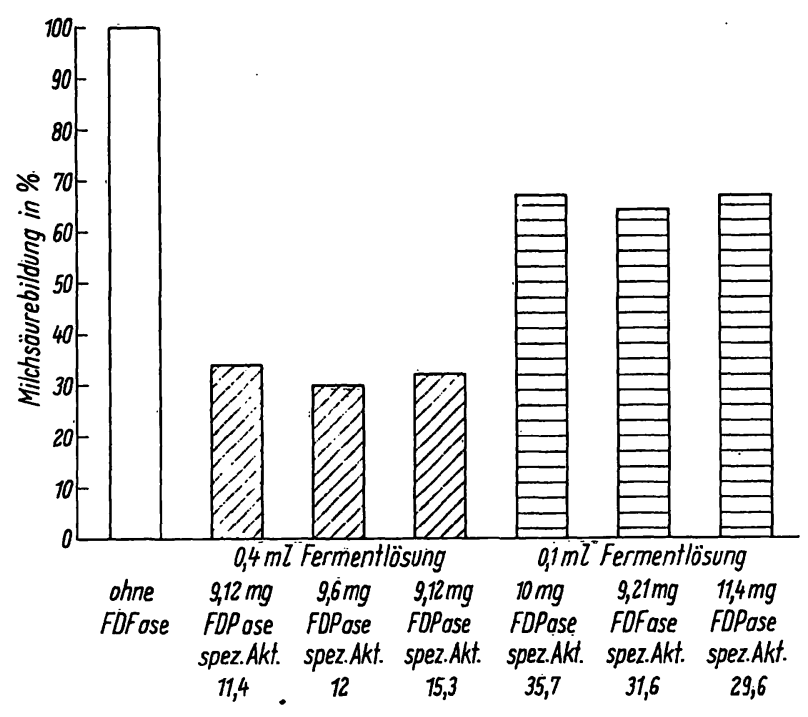

Abb. 1

Einfluß von FDP-ase auf die anaerobe Glykolyse von EhrlichAszites-Tumorzellen

Obwohl bei abnehmender Aktivität der verwendeten FDP-ase Präparate die Hemmung der anaeroben Glykolyse unterschiedlich war bzw. gleich blieb, konnte bei der letzten Aufbereitung der FDP-ase mit abnehmender Aktivität eine entsprechende Abnahme der Glykolysehemmung festgestellt werden. Wir glauben, daß es sich um eine reinere Fermentlösung gehandelt haben muß. Diesen Unterschieden sind unsere nächsten Untersuchungen gewidmet.

NaGh H. A. KREBs ergibt ein Zusatz von Milchsäure zu den Homogenaten von Taubenleber eine Neubildung von Zucker auf dem Wege der Umkehr der Glykolyse. Innerhalb der energetischen Verhältnisse untersuchte KREBS weiterhin den Einfluß von Adenosinmonophosphat („AMP"). Er erzielte dabei eine Hemmung der Zuckerneubildung bis zu $90 \%$. Dieses Ergebnis bewog uns, durch zusätzliche Gaben von AMP, ADP und ATP bei gleichzeitiger Anwesenheit von FDP-ase eine Einwirkung auf die Glykolyse der Ehrlich-Aszites-Zellen in der Warburg-Apparatur zu prüfen. Da ATP als energiereicher Phosphatdonator im Embden-Meyerhof-Abbauweg der Glykose bekannt ist, war es für uns interessant, inwieweit AMP, ADP und ATP und zusätzliche Gaben von FDP-ase auf den glykolytischen Stoffwechsel der Ehrlich-Aszites-Tumorzellen Einfluß nehmen.

\section{Methodik}

Das Inkubationsmedium für die Messung der anaeroben Glykolyse von Ehrlich-Aszites-Tumorzellen unter Zusatz von AMP, ADP und ATP in Abwesenheit von FDP-ase bestand aus einem RingerBikarbonat-Puffer vom $\mathrm{pH}=7,4$. Als Substrat wurde Glukose zugesetzt. Die Endkonzentration an Glukose betrug für jedes 
Abb. 2

Einfluß der Fermentalterung der FDP-ase und des unterschiedlichen Proteingehaltes auf die Glykolyschemmung von EhrlichAszites-Tumorzellen
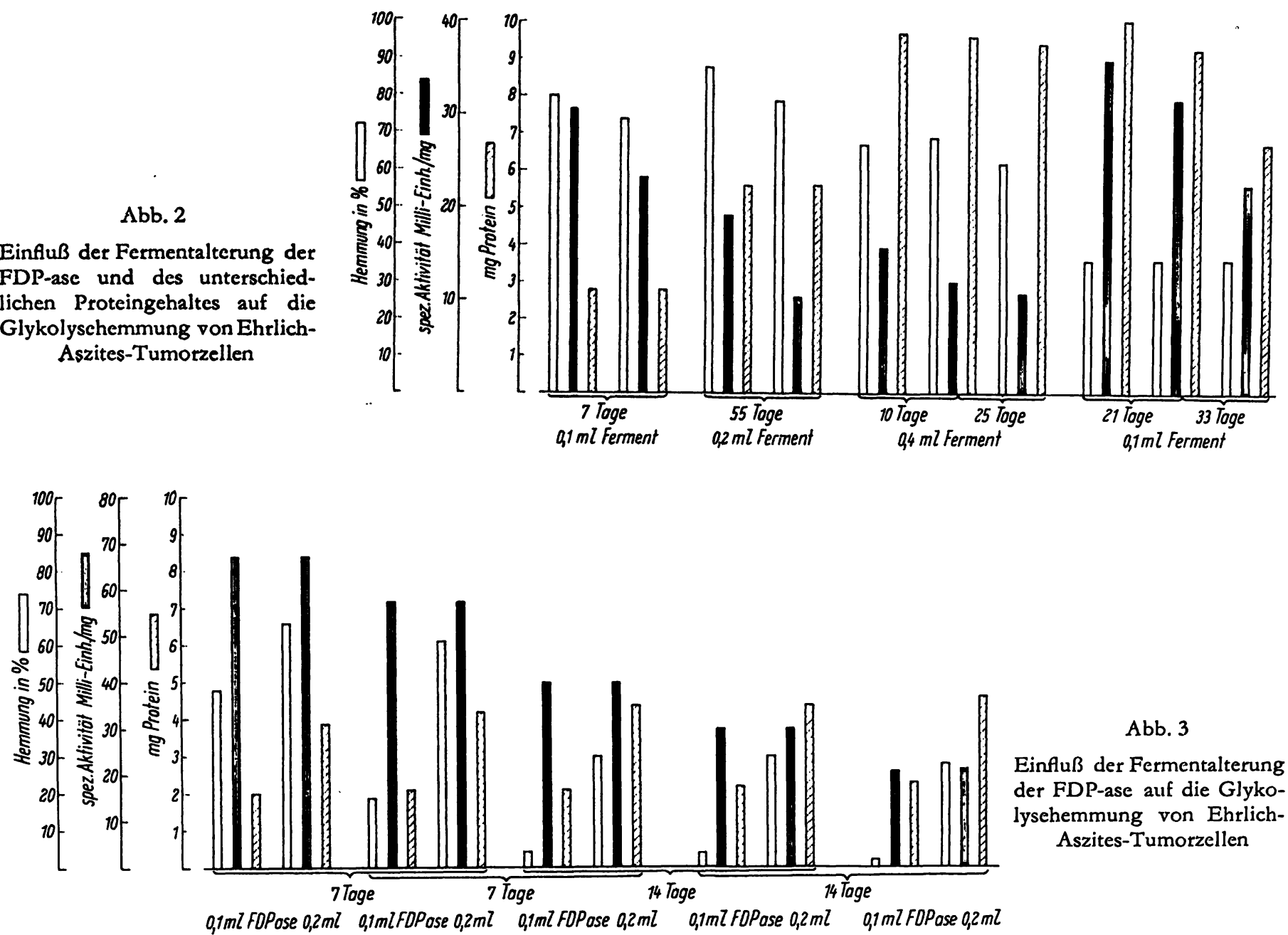

Einfluß der Fermentalterung der FDP-ase auf die Glykolysehemmung von EhrlichAszites-Tumorzellen

Gefäß 34,0 $\mu \mathrm{Mol}$. Die Ehrlich-Aszites-Tumorzellen wurden, wie bereits beschrieben, präpariert und in den Hauptraum der Gefäße pipettiert.

Die anaerobe Milchsäurebildung wurde nach BARKER und SUMmerson (10) bestimmt. Der Hauptraum der Gefäße enthielt je nach der Versuchsanordnung $0,5 \mathrm{ml}$ AMP- bzw. ADP- bzw. ATP-Lösung. Jede Versuchsanordnung umfaßte zwei Gefäße. Der Anhang der Gefäße enthielt $0,1 \mathrm{~m} l 20$-proz. Trichloressigsäure. Die im ersten Gefäß ablaufenden Stoffwechselvorgänge wurden zur Zeit $t=0$ durch Einkippen der Trichloressigsäure gestoppt. Der Stoffwechsel des zweiten Gefäßes wurde nach $60 \mathrm{Min}$. Inkubation bei $37^{\circ}$ im Wasserbad unterbrochen. Der Gasraum der Gefäße enthielt ein Gasgemisch von $5 \% \mathrm{CO}_{2}$ in $95 \% \mathrm{~N}_{2}$.

Bei den Versuchen mit AMP wurden 0,1 - bzw. 0,15 m/ FDP-ase in den Hauptraum der Gefäße zugegeben. Für die Versuche mit ADP und ATP wurden jeweils $0,4 \mathrm{ml}$ FDP-ase in den Hauptraum der Gefäße pipettiert. - Die spezifische Aktivität der für die Vcrsuche verwendeten Fermentlösung ist aus den jeweiligen Tabellen zu ersehen. Das Flüssigkeitsvolumen betrug für jedes Gefäß $3,1 \mathrm{ml}$.

Für die Messung der anaẹroben Glykolyse mit Nicotinsäureamid unter Zusatz von FDP-ase wurden je nach Versuchsanordnung $0,5 \mathrm{~m} l 0,02$ bzw. $0,03 m$ Nicotinsäureamidlösung sowie $0,25 \mathrm{~m} l$ FDP-aselösung in den Hauptraum der Gefäße pipettiert. Als Inkubationsmedium diente wieder ein Gemisch aus Ringer-Bikarbonat-Glukoselösung. Der Gastaum enthielt ein Gasgemisch von $5 \% \mathrm{CO}_{2}$ in $95 \% \mathrm{~N}_{2}$. Die spezifische Aktivität des Fermentpräparates wurde bei diesen Versuchen mit 26,91 Milli-Einh./mg Protein gemessen.

Die in den Tabellen angegebenen Werte für $\mu \mathrm{Mol}$ gebildete Milchsäure beziehen sich auf 6-8 mg Zelltrockengewicht/Ansatz. Die aufgeführten Zahlen stellen jedesmal Mittelwerte aus zwei Versuchsansätzen dar.

\section{Ergebnisse}

Die Tabelle 1 zeigt, daß zugesetzte AMP-Mengen unterschiedlicher Konzentration in Abhängigkeit von der spezifischen Aktivität der FDP-ase Differenzen im glykolytischen Abbauweg erkennen lassen. Die Ergebnisse zeigen, daß bei Einwirkung höherer AMP-Konzentrationen auf reine Asziteszellen die Glykolyse abnimmt. Die Wirkung der FDP-ase wird durch höhere AMPKonzentrationen obendrein verstärkt.

Die Tabelle 2 läßt erkennen, daß ein Zusatz von steigenden ADP-Mengen trotz unterschiedlicher spezifischer Aktivität der FDP-ase keinen deutlichen Einfluß auf die Glykolyse der Ehrlich-Aszites-Tumorzellen nimmt. Auf reine Asziteszellen ist ADP allein ebenfalls ohne Einfluß. Im Gegensatz hierzu hat der steigende Zusatz von ATP auf reine Asziteszellen eine glykolysefördernde Wirkung. In Kombination mit FDP-ase steigt die Glykolyse bei Zugabe von $12 \mu \mathrm{Mol}$ ATP/Ansatz um das Doppelte. Diese ATP-Wirkung kann auf eine Anregung der Hexokinasereaktion innerhalb des glykolytischen $\mathrm{Ab}-$ bauweges zurückgeführt werden, die eine verminderte FDP-ase Wirkung zur Folge hat.

PürTER und andere konnten nachweisen, daß die karzinostatische Wirkung von Zytostaticis auf Tumorzellen durch Nicotinsäureamid aufgehoben werden konnte. Dieser Effekt wurde auf eine Senkung des in der Tumorzelle erhöht gefundenen DPN bezogen (11). Dies bewog 
uns, den Einfluß steigender Nicotinsäureamidzugaben auf die FDP-ase Hemmwirkung bei glykolysierenden Ehrlich-Aszites-Tumorzellen zu prüfen. Die Tabelle 4 läßt erkennen, daß Nicotinsäureamid die FDP-ase Hemmwirkung auf die Glykolyse nicht aufheben kann. Auf reine Asziteszellen hat Nicotinsäureamid allein ebenfalls keinen Einfluß.
Wir sind zu Dank verpflichtet:

der Arbeitsgemeinschaft für Krebsbekämpfung im Lande Nordthein-Westfalen, Sitz Bochum, Ruhrknappschaft, für die Ermöglichung dieser Untersuchung;

den Bayer-Werken Leverkusen für die Uberlassung der FDP-aseFermentlösung;

Fräulein ERIKA WIEMERsLAGe für ihre technische Assistenz.

Tab. 1

Anaerobe Glykolyse von Ehrlich-Aszites-Tumorzellen bei Einwirkung steigender AMP Konzentrationen unter Zusatz von Fructose1,6-diphosphatase in Ringer-Bikarbonat-Puffer mit 34,0 $\mu \mathrm{Mol}$ Glucose/Ansatz

\begin{tabular}{|c|c|c|c|c|c|c|}
\hline \multirow{3}{*}{$\begin{array}{c}\text { Zugefügtes } \\
\text { AMP } \\
(\mu \mathrm{Mol})\end{array}$} & \multirow{3}{*}{$\begin{array}{c}\text { Zugefügte } \\
\text { Fermentlösung: } \\
0,1 \mathrm{bzw} .0,15 \mathrm{ml} \\
\text { Proteinmenge in (mg) }\end{array}$} & \multirow{3}{*}{$\begin{array}{c}\text { Spezifische } \\
\text { Aktivität } \\
\text { der FDP-ase }\end{array}$} & \multicolumn{4}{|c|}{ Milchsäurebildung (in $\mu \mathrm{Mol}$ ) von Asziteszellen in $60 \mathrm{Min}$. } \\
\hline & & & Ohne Zusatz & Zusatz von & Zusatz von & Zusatz von \\
\hline & & & & AMP & FDP-ase & FDP-ase + AMP \\
\hline 3 & 2,05 & 64 & \multirow{2}{*}{15,55} & 15,93 & \multirow{2}{*}{6,63} & 6,38 \\
\hline 6 & 2,05 & 64 & & 14,71 & & 6,66 \\
\hline 9 & 2,05 & 64 & \multirow{2}{*}{16,43} & 15,21 & \multirow{2}{*}{6,92} & 6,67 \\
\hline 12 & 2,05 & 64 & & 15,41 & & 6,29 \\
\hline 15 & 3,13 & 57,5 & \multirow{2}{*}{15,13} & 12,49 & \multirow{2}{*}{9,71} & 7,20 \\
\hline 20 & 3,13 & 57,5 & & 11,58 & & 7,22 \\
\hline 25 & 3,13 & 57,5 & \multirow{2}{*}{15,01} & 11,03 & \multirow{2}{*}{9,68} & 6,82 \\
\hline 30 & 3,13 & 57,5 & & 10,95 & & 6,52 \\
\hline
\end{tabular}

Tab. 2

Anaerobe Glykolyse von Ehrlich-Aszites-Tumorzellen bei Einwirkung steigender ADP Konzentration unter Zusatz von Fructose1,6-diphosphatase in Ringer-Bikarbonat-Puffer mit 34,0 $\mu \mathrm{Mol}$ Glucose/Ansatz

\begin{tabular}{ccccccc}
\hline $\begin{array}{c}\text { Zugefügtes } \\
\text { ADP } \\
(\mu \mathrm{Mol})\end{array}$ & $\begin{array}{c}\text { Zugefügte FDP-ase- } \\
\text { Lösung }=0,4 \mathrm{~m} l \\
\text { Proteinmenge in (mg) }\end{array}$ & $\begin{array}{c}\text { Spezifische } \\
\text { Aktivität } \\
\text { det FDP-ase }\end{array}$ & $\begin{array}{c}\text { Milchsäurebildung (in } \mu \mathrm{Mol}) \text { von Asziteszellen in } 60 \text { Min. } \\
\text { Ohne Zusatz }\end{array}$ & $\begin{array}{c}\text { Zusatz von } \\
\text { ADP }\end{array}$ & $\begin{array}{c}\text { Zusatz von } \\
\text { FDP-ase }\end{array}$ & $\begin{array}{c}\text { Zusatz von } \\
\text { FDP-ase + ADP }\end{array}$ \\
\hline 3 & 8,64 & 17,60 & 16,01 & 16,23 & 6,00 & 6,43 \\
6 & 8,64 & 17,60 & 15,86 & 15,54 & 5,95 & 6,21 \\
9 & 9,60 & 17,60 & 15,73 & 16,15 & 6,09 & 6,83 \\
12 & 9,52 & 15,10 & 15,98 & 15,43 & 5,34 & 5,52 \\
\hline
\end{tabular}

Tab. 3..

Anaerobe Glykolyse von Ehrlich-Aszites-Tumorzellen bei Einwirkung steigender ATP Konzentrationen unter Zusatz von Fructose1,6-diphosphatase in Ringer-Bikarbonat-Puffer mit 34,0 $\mu \mathrm{Mol}$ Glucose/Ansatz

\begin{tabular}{|c|c|c|c|c|c|c|}
\hline \multirow{2}{*}{$\begin{array}{l}\text { Zugefügtes } \\
\text { ATP } \\
(\mu \text { Mol })\end{array}$} & \multirow{2}{*}{$\begin{array}{l}\text { Zugefügte FDP-ase- } \\
\text { Lösung }=0,4 \mathrm{~m} l \\
\text { Proteinmenge in (mg) }\end{array}$} & \multirow{2}{*}{$\begin{array}{l}\text { Spezifische } \\
\text { Aktivität } \\
\text { der FDP-ase }\end{array}$} & \multicolumn{4}{|c|}{ Milchsäurebildung (in $\mu \mathrm{Mol}$ ) von Asziteszellen in $60 \mathrm{Min}$. } \\
\hline & & & Ohne Zusatz & $\begin{array}{l}\text { Zusatz von } \\
\text { ATP }\end{array}$ & $\begin{array}{l}\text { Zụsatz von } \\
\text { FDP-ase }\end{array}$ & $\begin{array}{c}\text { Zusatz von } \\
\text { FDP-ase + ATP }\end{array}$ \\
\hline 3 & 8,64 & 17,60 & 16,23 & 19,39 & 5,13 & 5,06 \\
\hline 6 & 8,64 & 17,60 & 15,96 & 19,20 & 5,86 & 6,40 \\
\hline 9 & 9,60 & 17,60 & 15,03 & 15,79 & 5,53 & 8,89 \\
\hline 12 & 9,52 & 15,10 & 15,78 & 17,20 & 5,95 & 11,66 \\
\hline
\end{tabular}

Tab. 4

Anaerobe Glykolyse von Ehrlich-Aszites-Tumorzellen bei Einwirkung von Fructose-1,6-diphosphatase und Nicotinsäureamid in RingerBikarbonat-Puffer mit 34,0 $\mu \mathrm{Mol}$ Glucose/Ansatz. Spez. Aktivität der FDP-ase: 26,91. Pro Ansatz wurden 0,25 ml FDP-ase-Lösung $=5,70 \mathrm{mg}$ Protein eingesetzt

\begin{tabular}{|c|c|c|c|c|c|}
\hline \multirow[b]{2}{*}{ Ohne Zusatz } & \multicolumn{4}{|c|}{ Milchsäurebildung (in $\mu \mathrm{Mol}$ ) von Asziteszellen in $60 \mathrm{Min}}$. & \multirow[b]{2}{*}{$\begin{array}{l}\text { Zusatz von FDP-ase } \\
+0,03 m \text { Nicotin- } \\
\text { säureamidlösung }\end{array}$} \\
\hline & $\begin{array}{c}\text { Zusatz von } 0,02 \mathrm{~m} \\
\text { Nicotinsäureamid- } \\
\text { lösung }\end{array}$ & $\begin{array}{c}\text { Zusatz von } 0,03 \mathrm{~m} \\
\text { Nicotinsäureamid- } \\
\text { lösung }\end{array}$ & Zusatz von FDP-ase & $\begin{array}{l}\text { Zusatz von FDP-ase } \\
+0,02 m \text { Nicotin- } \\
\text { säureamidlösung }\end{array}$ & \\
\hline 16,40 & 16,57 & 16,02 & 9,82 & 9,79 & 9,72 \\
\hline 16,15 & 16,24 & 15,78 & 9,77 & 9,71 & 9,66 \\
\hline
\end{tabular}




\title{
Literatur
}

1. Lürrs, W., a) Arch. Geschwulstforsch. 17, 222 (1961); b) Z. Naturforsch. 166, 254 (1961); c) Krebsforschung und Krebsbekämpfung, 4. Sonderband, S. 111, Urban \& Schwarzenberg, München/Berlin (1961). - 2. KREBS, H. A., 13. Nobelpreisträgertagung, Lindau, 1.-5. 7. 1963, Proc. Roy. Soc. Edinburgh, sert. B 159, 545 (1964). - 3. Warburg, O., Uber den Stoffwechsel der Tumoren, Springer-Verlag Berlin (1926). - 4. WeBER, G. und A. Cantero, Science 120, 851 (1954). - 5. a) Weber, G. und J. Ashmore, Exper. Cell. Res. 14, 226 (1958); b) Weber, G. und A. Cantero, Cancer Res. 19, 763 (1959). - 6. Lohmann, K., Biochem. Z. 262, 137 (1933). - 7. Roche, J. und S. BouchILlOux, Bull. Soc. Biol. 32, 739 (1950). - 8. LüHRs, W., Therap. Gegenw. 99, 375 (1960). - 9. Lewis, C., Mokrasch und R. W. MCGilvery, J. biol. Chemistry 221, 221 (1956). - 10. Barker, S. B. und W. H. Summerson, J. biol. Chemistry 138, 535 (1941). - 11. Pütrer, J., Therap. (Bayer) 32, 255 (1960).

Professor Dr. med. W. Lührs 8183 Rottach-Egern

Ringbergstr. 30

\section{Anaerobe Glykolyse bei Ehrlich-Aszites-Tumorzellen}

\author{
2. Mitteilung: Beeinflussung durch Antidiabetika
}

Von W. Lührs und K. (hrometzKA ${ }^{1}$ )

\begin{abstract}
Aus dem Sanatorium "Bergfrieden" - Rottach-Egern der Arbeitsgemeinscbaft fïr Krebsbekämpfung, Sitz Bocbum Rubrknappschaft (Chefarzt: Prof. Dr. W. Liibrs)
\end{abstract}

(Der Schriftleitung zugegangen am 16. Dezember 1963)

\begin{abstract}
Zugabe von Insulin führt sowohl bei Aszites-Tumorzellen als auch bei humanem Karzinomgewebe bei gleichzeitiger Zugabe von Fruktose-1,6-Diphosphat zu einem Anstieg der anaeroben Glykolyse. Im Gegensatz dazu fand sich bei Zugabe von Sulfonylharnstoffen (Rastinon) eine deutliche Hemmung der Glykolyse bei Ehrlich-AszitesZellen, und zwar einmal bei Zugabe von Glukose allein und zum andern bei zusätzlicher Zugabe von Fruktose-1,6Diphosphat. Die Biguanide waren in sämtlichen gleichartigen Versuchsansätzen wirkungslos.

The simultaneous addition of insulin and fructose-1,6-diphosphate causes an increase of anaerobic glycolysis in both asites tumour cells and human carcinoma tissue. On the other hand, the addition of sulphonylurcas (rastinone) caused a marked inhibition of glycolysis in Ehrlich ascites cells, once with the addition only of glucose, but otherwise with the addition also of fructose-1,6-diphosphate. The biguanides were inactive in several similar experiments.
\end{abstract}

Mögliche Beeinflussung der Glukoneogenese führten uns (1) $\mathrm{zu}$ Untersuchungen mit den heute angewandten Antidiabetika. Diese Substanzen bedingen eine bessere Verwertung erhöhter Glukosemengen im Serum, z. B. von Diabetikern. Viele ältere und neuere klinische Arbeiten zeigen immer wieder, daß eine Verbindung zwischen Diabetes und Karzinom nicht häufig gefunden wird. So berichtete unter anderen WERNER (2), daß die Auswertung von über 25000 Sektionsfällen eine signifikant geringere Häufigkeit diabetischer Karzinomträger gegenüber den Nichtdiabetikern ergeben hat. Rockstrof und Schröter (3) fanden bei der Auswertung von etwa 23000 Sektionsprotokollen eine signifikante negative Syntropie der Krebshäufigkeit beim Diabetes mellitus.

Wenn ich aus meiner eigenen Erfahrung sprechen darf, so habe ich bei etwa 20000 bisher beobachteten Krebsfällen nur beim hormonabhängigen Mammâ-Karzinom in etwas häufigerer Kombination einen Diabetes gefunden. Bei den epithelialen Karzinomen anderer Lokalisation waren bei diesem Krankengut nicht mehr als 20 Einzelfälle in Kombination mit einem Diabetes.

1) Teilergebnisse dieser Arbeit wurden auf dem Deutschen Krebskongre $B 1963$ in Mainz vorgetragen.
Für den Wirkungsmechanismus des Insulins wird die Notwendigkeit einer Glukose-Verwertung in Muskulatur, Fettgewebe und Leber hervorgehoben. Die weiteren Wirkungen, wie Glykogen-, Fett- und Proteinsynthese sind wahrscheinlich nur Folgen der Glukoseutilisation. Die Insulinwirkung wird häufig als ein Membraneffekt angesehen, wobei Insulin den Eintritt der Glukose in die Zelle beschleunigt (Levine und Goldstein, 4). Hierbei scheint eine Wirkung auf die Hexokinase nicht unwesentlich zu sein. Denn andere Autoren messen der Aktivierung der Glukosephosphorylierung innerhalb der Zelle, also der Wirkung der Hexokinasereaktion eine mindest ebenso große Bedeutung bei (5). In der Leberzelle, deren Membran für die Glukose wahrscheinlich frei durchlässig ist, kommt ein Membraneffekt nicht in Frage. Das Insulin könnte vorwiegend einen adaptiven Effekt auf Enzyme der Leberzelle haben, wodurch der verzögerte Wirkungseintritt erklärt wird (6). Allerdings ist gerade in den letzten Jahren eine aus-

Abkiirzungen :

„F-6-Pc $=$ Fruktose-6-phosphat „FDP“c = Fruktose-1,6-diphosphat „FDP-ase" $=$ Fruktose-1,6-diphosphatase 